\title{
The CVT's Speed Ratio Control Research When the Vehicle Bending at High Speed
}

\author{
Xinhua Yang* \\ College of Vehicle Engineering, Chongqing University of Technology, No.69 Hongguang Avenue, Banan District, Chongqing, P. \\ R. China \\ ${ }^{*}$ Corresponding author
}

\begin{abstract}
When the speed ratio of the vehicle equipped with CVT changes rapidly, the acceleration of the vehicle will change synchronously, that means to exert an extra force on the driving tires. Using this feature, the desired results can be obtained in certain specific conditions, such as vehicle cornering at high speed. The models of multi DOF vehicle and CVT transmission were established to study the rapid changes in the turning process of the vehicle through the CVT ratio control. This paper introduces a method based on safety factor of CVT speed ratio control strategy. The simulation results show that if the driver fails to effectively reduce vehicle speed during high-speed cornering. As the speed ratio of CVT increasing rapidly, and the backward force can be acquired on the driving wheel, so that the vehicle can keep straight in a short time while reducing vehicle speed. The results of this paper can achieve better control results if the engine fuel injection control is combined.
\end{abstract}

Keywords-CVT; speed ratio; control strategy; high speed cornering; multi DOF vehicle model

\section{INTRODUCTION}

Because the speed ratio of the metal belt Continuously Variable Transmission(CVT) changes continuously, and the power is uninterruptable at the mean time, many ideal control effects can be obtained, and the engine running more stable and economically. Therefore, the CVT's control strategies are recognized as one of the key technologies in this field. Researchers all over the world have carried out a great deal of research on the control strategy of CVT. The study of CVT control technology is mainly focused on the speed ratio control to increase the engine's output torque and decrease it's fuel consumption, through controlling engine speed according to the engine power or economy operation line, and how to achieve the target operating condition instantaneously. ${ }^{[1][2][3]}$

From the research available, the main study focuses are on the analysis of control strategy, and different control strategies adopted for different working conditions. Because of the complex working conditions encountered in vehicle operation, the control strategies research on CVT speed ratio when cornering is very rare. The author in the foregoing theoretical research, put forward the mathematical expressions between the vehicle acceleration and CVT speed ratio's variance ratio. It not only showed the relationship between speed and acceleration, but also guided the strategy analysis of CVT speed ratio control, and revealed the relationship between ratio change rate and vehicle acceleration ${ }^{[4]}$. Using these characteristics of CVT speed ratio, we can control vehicle acceleration in a short time, so that even when the output power of the engine is not reduced, the vehicle can achieve short time deceleration or acceleration. For example, when the driver driving a car enters the bend at high speed, if there is no proper deceleration, the vehicle will be tilting or even overturning. The proper intervention of the transmission at this time helps the vehicle to slow down and help the vehicle to recover at the time of the cornering. This paper presents a basic control theory and control method of active vehicle safety technology based on CVT speed ratio control, which lays a theoretical foundation for developing CVT based on this technology.

\section{MATHEMATICAL MODEL}

The basic assumptions are as follows: only considering the longitudinal air resistance and the air resistance of other direction is neglected; front and rear wheel tread are equal; and no road level fluctuation. The force analysis and dynamic equation of vehicle can be referred to reference literature ${ }^{[5]}$.

For the power transmission system, the differential equations of the driving and driven axes are listed as follows:

$$
\left\{\begin{array}{c}
I_{1} \ddot{\theta}_{1}+C_{1} \dot{\theta}_{1}+K_{1}\left(\theta_{1}-i_{\mathrm{g}} \cdot \theta_{2}\right)=T_{e}-T_{c v t} \\
I_{2} \ddot{\theta}_{2}+C_{2} \dot{\theta}_{2}+K_{2}\left(\theta_{2}-i_{0} \cdot \theta_{t}\right)=i_{g} \cdot T_{c v t}-T_{f}
\end{array}\right.
$$

Symbolic description: 'I' represents rotary inertia, 'C' represents damping, ' $K$ ' represents rigidity, ' $T$ ' represents torque, 'i' represents speed ratio.

The subscript description: ' 1 ' represents the input shaft(the driving shaft); ' 2 ' represents the output shaft(the driven shaft); ' $e$ ' represents the engine; ' $f$ ' stands for the resistance; 'cvt' represents the continuously variable transmission; ' $t$ ' stands for the transmission output shaft.

Symbolic description : $\omega$-rotation speed, r/min; $\theta$ rotation angle, rad; $i_{\mathrm{g}}$-speed ratio of CVT.

According to the transmit relationship of driving and driven axes of CVT, the following formula is set up.

$$
\omega_{1}=i_{\mathrm{g}} \cdot \omega_{2} \text {, that is } \dot{\theta}_{1}=i_{g} \cdot \dot{\theta}_{2}
$$


It's differential is derivation as follows.

$$
\ddot{\theta}_{1}=\dot{i}_{g} \cdot \dot{\theta}_{2}+i_{g} \cdot \ddot{\theta}_{2}
$$

Merge (1), (2) and (3), the following formula (4) is derived.

$$
\begin{aligned}
& \left(I_{1} \cdot i_{\mathrm{g}}{ }^{2}+I_{2}\right) \ddot{\theta}_{2}+\left[C_{2}+i_{g}\left(I_{1} \cdot \dot{i}_{g}+C_{1} \cdot i_{g}\right)\right] \dot{\theta}_{2}+ \\
& K_{1} \cdot i_{g}\left(\theta_{1}-i_{g} \theta_{2}\right)+K_{2}\left(\theta_{2}-i_{0} \theta_{t}\right)=i_{g} \cdot T_{e}-T_{f}
\end{aligned}
$$

In this formula, the variation rate of CVT speed ratio is equivalent to the extra damping in the motion equation, so the moving state of the drive shaft can be controlled by the rate of change of speed ratio. Ignoring the stiffness $\mathrm{K}$ and damping $\mathrm{C}$ in (4), the following formula (5) can be obtained.

$$
\left(I_{1} \cdot \dot{i}_{g}^{2}+I_{2}\right) \cdot \ddot{\theta}_{2}=T_{e} \cdot i_{g}-T_{f}-i_{g} \cdot I_{1} \cdot \dot{i}_{g} \cdot \dot{\theta}_{2}
$$

From the above formula, it can be seen that when the speed ratio of CVT changes rapidly, the direction and size of the change will cause the change of the output axis's rotation acceleration. Please refer to [6] for detailed analysis.

Using this characteristic, the vehicle can obtain extra acceleration or deceleration under specific conditions. Such as when cornering at high speed, the quick increase of speed ratio makes the vehicle slow down for a short time and then accelerates out the bending.

\section{CONTROL STRATEGY}

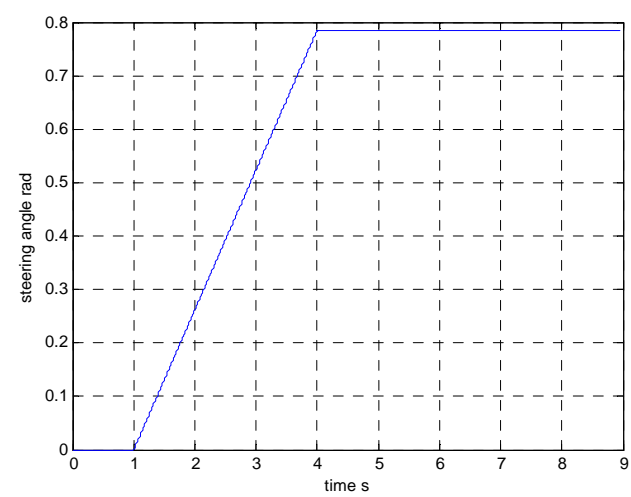

FIGURE I. THE STEERING ANGLE OF DRIVING TYRE

When the vehicle enters the bend, for the high speed and centrifugal force, if the driver does not carry out the reasonable braking operation, the dangerous working conditions of roll over may appear. [7] It is believed that when the vehicle's lateral acceleration reaches $0.4 \mathrm{~g}$, the human body is not feeling well, and increasing the lateral acceleration may lead to the risk of vehicle rollover. The threshold of vehicle rollover is related to the automobile models, and the roll over threshold of a car is about $0.7 \mathrm{~g} \sim 1.2 \mathrm{~g}$ at the step steering process. Therefore, we take the lateral acceleration of $0.4 \mathrm{~g}$ as the threshold for the control system to participate in the vehicle dynamics control. From the results of the previous model test, the steering angle greater and the speed higher, the dangerous conditions appear easier. When the steering wheel turns in accordance with the laws shown in Figure I, the relationship between the vehicle speed and the maximum turning angle of the steering wheel is illustrated. In Figure II, the area on the top part of the curve is the dangerous driving area, and the one under the curve is safe area. The line was drawn according to the roll over threshold. When the vehicle is approaching the limit condition, the deceleration operation should be carried out ahead of time, so as to prevent the occurrence of danger.

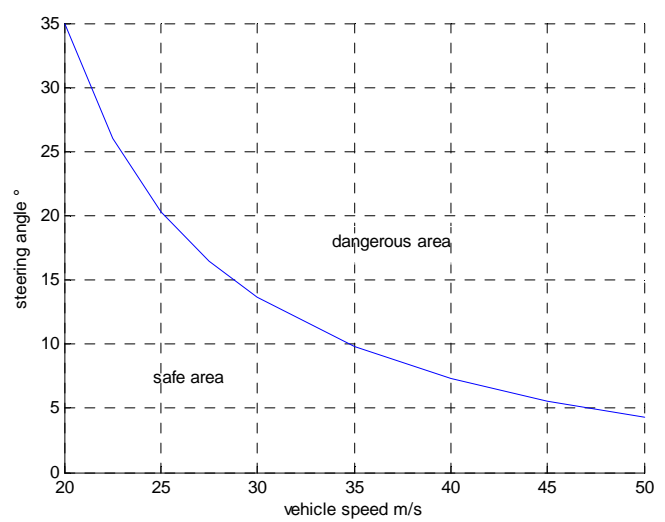

FIGURE II. THE RELATIONSHIP BETWEEN THE VEHICLE SPEED AND THE STEERING ANGLE

The speed ratio control logic in bending is that when the driver turning the steering wheel, the variation rate of steering tires' slip angle is larger than zero, and after a short time, it would not exceed the allowable values in FIGURE II. This logic can be expressed as the following mathematic formulas.

$$
\left\{\begin{array}{l}
|\alpha|+\frac{d|\alpha|}{d t} \cdot \Delta \mathrm{t}>\alpha_{\max } \\
\frac{d|\alpha|}{d t}>0
\end{array}\right.
$$

$$
\alpha \text { is the steering wheel's slip angle. }
$$

The basic idea of this strategy is to determine whether the current steering wheel angle increases or not, and meanwhile whether it will exceed the set safety angle in the next time according to the speed of the current rotation. This is a prediction based on the current angle change rate for the future angle.

\section{SiMULATION RESULTS}

According to the mathematical model and control strategy deduced above, a simulation model based on MATLAB/SIMULINK was set up. The vehicle speed was set to $25 \mathrm{~m} / \mathrm{s}$. The driver turns the steering wheel, and the slip angle of the steering wheel changes. As shown in Figure I, the driver does not carry out the deceleration operation. That means the throttle is keep $100 \%$ and there is no brake operation. 
In order to compare the influence of CVT speed ratio on this process, Test I is set as the CVT's speed ratio maintain minimum and unchanged. Test II determines the speed ratio according to the control logic, and performs fast changing. The comparison of the speed ratio of the two tests is drawn in Figure III. In the Test II, the speed ratio increases rapidly to the maximum, and then quickly goes back to the minimum.

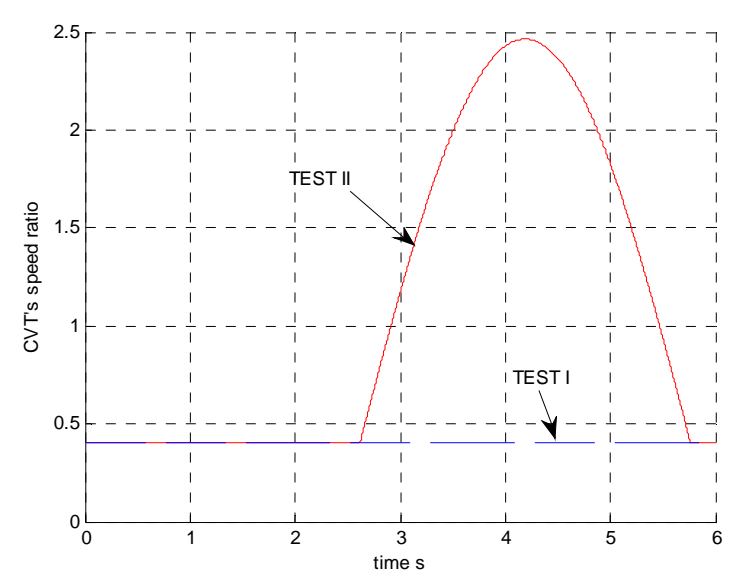

FIGURE III. THE COMPARISON OF THE SPEED RATIO

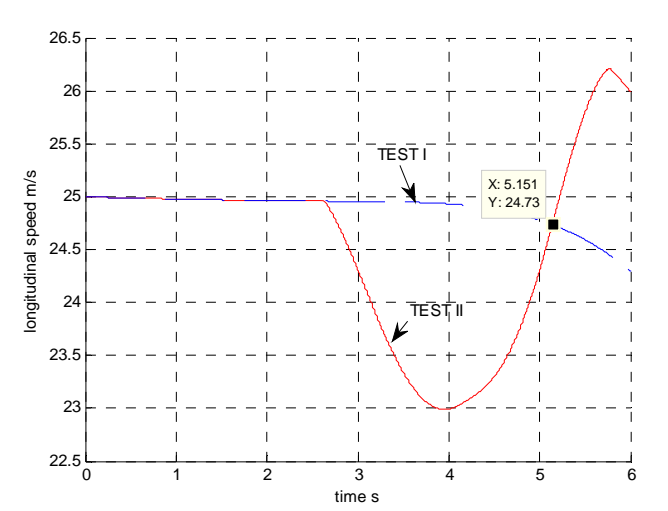

FIGURE IV. THE COMPARISON OF THE LONGITUDINAL SPEED

FIGURE IV shows the change in the longitudinal speed of the vehicle during this process. In the test I, as the vehicle enters the bend, the longitudinal velocity decreases with the deflection of the steering wheel. In the Test II, when the system is about 2.6 seconds, the control system judged that the vehicle will enter the dangerous working condition, so the speed ratio increased rapidly, which caused the transmission system to suffer the braking torque and slow down. The speed of the car is reduced by about $2 \mathrm{~m} / \mathrm{s}$ to $23 \mathrm{~m} / \mathrm{s}$. After that, with the rapid recovery, the vehicle speed increased. At the time about 5.15 seconds, the longitudinal speed of the test condition II exceeded the test condition I, that means the vehicle runs faster. It is because that the speed ratio is larger, and the transmission output torque is amplified.

FIGURE V contrasts the lateral acceleration of the two tests. In the Test I, as the driver turns the steering wheel, the lateral acceleration increases as the vehicle deflects. In the test of II, due to the rapid change of CVT's speed ratio, the driving force of the driving wheel disappears, and it turns into a backward braking force, which causes the vehicle to reverse in the side direction. When the speed ratio reaches the maximum at 4.1 seconds and then decreases as shown in Figure III , the axes restores the driving energy, and the vehicle begins to deflect which can be seen from the Test II curve in Figure IV. At about 5 seconds, the lateral acceleration of the Test II exceeds the test I. The peak is reached after 5 seconds, which has exceeded the safe lateral acceleration $0.4 \mathrm{~g}$. This shows that if the power output of the engine is always maintained, the speed ratio control strategy adopted in Test II can not only achieve the purpose of reducing risk, but also cause greater lateral acceleration, which makes the vehicle easier to roll over.

The lateral velocities of the two tests are compared in the Figure VI. It can be seen from the graph of Test II that in the period from 2 seconds to 4 seconds, the vehicle has no lateral deflection. Then the lateral velocity began to increase, and the lateral velocity of Test II exceeded the one of Test I after 5 seconds.

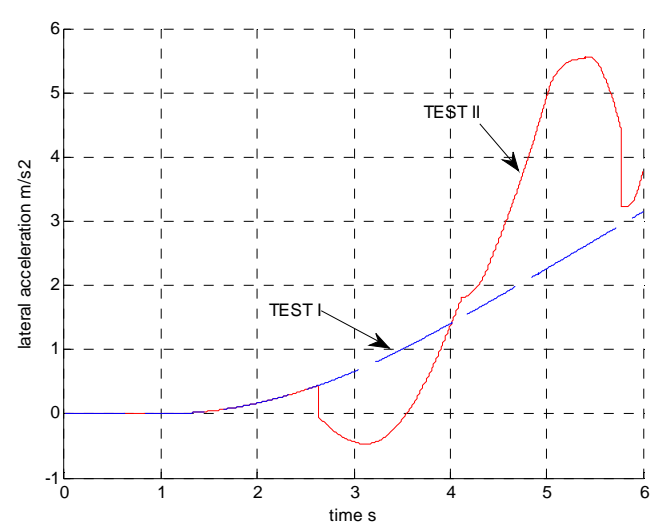

FIGURE V. THE COMPARISON OF LATERAL ACCELERATION

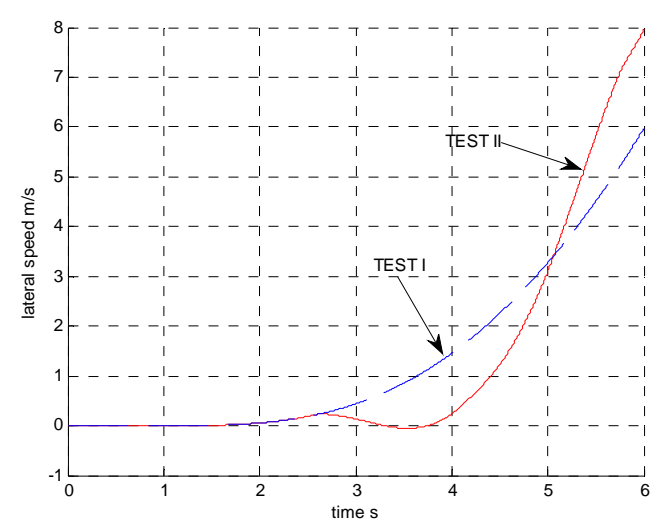

FIGURE VI. THE COMPARISON OF THE LATERAL SPEED

FIGURE VII is the contrast of vehicle tracks between the two Tests. From the graph, the trajectory of Test II is difference with the one of Test I. In Test II, the vehicle almost keeps a straight movement within 50-100 meters after turning, and then it has a quicker turn and a smaller turning radius than Test I. 


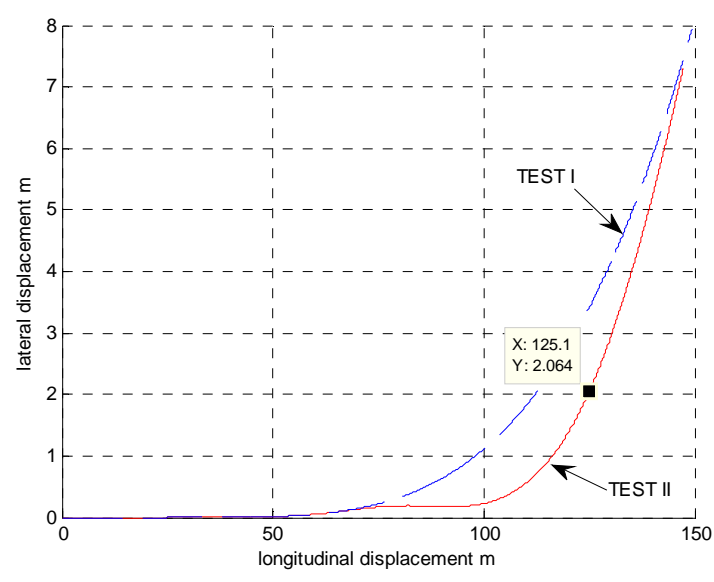

FIGURE VII. THE COMPARISON OF THE TRACKS

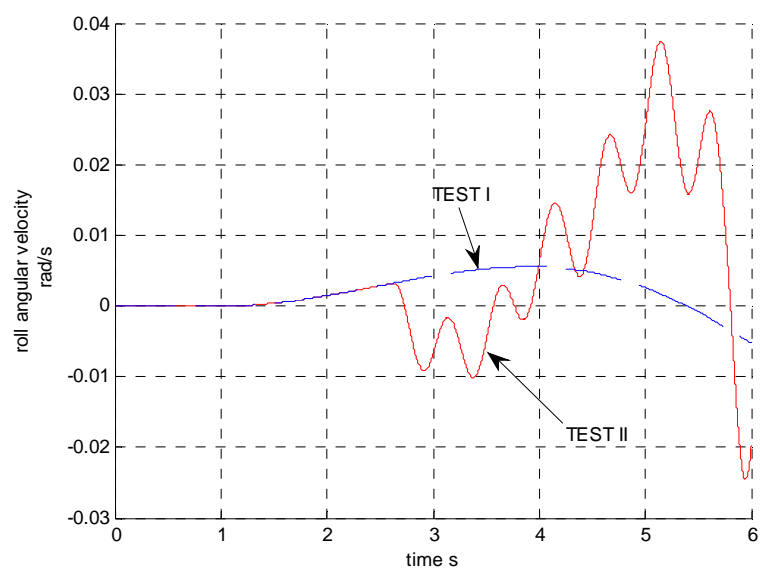

FIGURE VIII. THE COMPARISON OF ROLL ANGULAR VELOCITY

Finally, the roll velocities of the vehicle are compared, as shown in figure VIII. In Test I, the driver normally turns, the vehicle roll velocity fluctuates little. The test II presents a fluctuation with higher frequency and larger amplitude. This is related to the violent change of the vehicle's lateral acceleration. The comparison results show that the rapid change of CVT speed ratio will lead to the decrease of comfort.

From the simulation comparison chart above, the CVT ratio control strategy put forward in this paper which includes a rapid increase and fast recovery ratio can reduce the vehicle speed in a short period of time, as a result the driver can obtain about two seconds to response. During this period, the driver can take corresponding operations to maintain safe driving, such as braking, holding up the steering wheel etc.. But after 2 seconds, the kinetic energy stored in the powertrain will be released. The higher the speed and the greater the lateral acceleration, the more dangerous the vehicle will be. In this case, other body control systems need to be combined to help the driver maintain a safe running state, for example the Electronic Stability Program.

Another possible control strategy is to rapidly increase the speed ratio to the maximum speed ratio, and keep the speed ratio unchanged. The engine stops fuel injection meanwhile. Because of the large speed ratio of the transmission, the engine speed is pulled up. As the result of this, the engine's braking torque is also large, this can also help the vehicle speed down. How to combine these control systems is still needed to be further studied.

\section{CONCLUSIONS}

The paper makes a preliminary study on the control strategy of CVT when the vehicle turns a corner at high speed. The possibility of controlling vehicle motion state by changing the speed ratio rapidly when the vehicle enters the corner at high speed was studied through a multi degree of freedom vehicle and CVT transmission combined system model. The simulation results show that when the vehicle cornering at high speed, the rapid change of CVT speed ratio will lead to a great change of vehicle motion state. And if the speed ratio increase rapidly, the vehicle speed will be reduced and maintain smaller vehicle deflection in a short time. But then the vehicle will speed up the bending and the tilting trend is increasing. Although the control strategy can obtain about 2 seconds response time for the driver, if the effective measures can not be taken, the vehicle will then enter a more dangerous condition. This needs to be combined with engine control and other control systems in the body to achieve better safety over bending.

\section{ACKNOWLEDGMENT}

My works was supported by Scientific and Technological Research Program of Chongqing Municipal Education Commission (Grant No.KJ1500936, KJ1400935).

\section{REFERENCES}

[1] C. Chan, D. Yang, T. Volz, et al. System Design and Control Considerations of Automotive Continuously Variable Transmissions, SAE Paper 840048

[2] Kim, Hyunsoo, Hanlim, et al. Metal Belt CVT and Engine Optimal Operation by PWM Electro-Hydraulic Control. International conference on Continuously Variable Power Transmissions CVT '96, Yokohama Proceedings, Sept. 11-12, 1996, Conf. Pub. No. 304

[3] Hong, Chewun. An Automotive Dynamic Performance Simulator for Vehicular Powertrain System Design. Int. J. of Vehicle Design, 1995, vol.16, pp. 264-281

[4] N. Srivastava, I. Haque. A review on belt and chain continuously variable transmissions (CVT): Dynamics and control. Mechanism and Machine Theory,2008, vol. 44, pp. 19 41,

[5] Yan Ruilei, The Simulation and Analysis of Fourteen-Degree-Freedom Vehicle Dynamic Model, M.D thesis, Hunan Universtiy, Changsha, 2014

[6] Xinhua Yang, Electro-Mechanical Continuously Variable Transmission Control System and Control Strategies, PH.D thesis, Huazhong University of Science and Technology, Wuhan , 2010

[7] Zhisheng Yu, Automobile Theory, $5^{\text {th }}$ ed., China Machine Press, China, 2015 